\title{
A symptomatic sesamoid bone in the popliteus muscle (cyamella)
}

\author{
Jan Philipp Benthien · Alexander Brunner
}

Received: 9 February 2010/Accepted: 19 October 2010/Published online: 21 November 2010

(C) Springer-Verlag 2010

\begin{abstract}
Sesamoid bones of the popliteus muscle, also called cyamellae, are common in primates but rare in humans. They reside as accessory bones in the tendon itself or in the intersection between tendon and bone. They should be clearly distinguished from osteochondral flakes, periosseuos calcifications, osteophytes and the fabella, a common sesamoid bone of the knee. In this case, we report a 25-year-old male with posterior lateral knee pain related to the popliteus tendon where a corresponding cyamella could be demonstrated on X-rays and MRI scans. The clinical findings may be related to the cyamella. Diagnosis and treatment of this rare pathology are discussed.
\end{abstract}

Keywords Cyamella $\cdot$ Sesamoid bone $\cdot$ Knee joint

\section{Introduction}

Small accessory bones residing in tendons and muscles are also known as sesamoid bones [1-3]. Sesamoid bones are small accessory bones usually embedded in tendons or muscles, typically near joints. They seem to be part of the foetal skeletal development and are regressive with a tendency to fuse in adults. Sesamoid bones may be asymptomatic or symptomatic, causing considerable pathology [2]. They may perform bony or cartilaginous pseudo-

J. P. Benthien $(\bowtie)$

Department of Orthopaedic Surgery, Hip, Knee and Prosthetics

Division, University of Basel, Spitalstr. 21, 4031 Basel,

Switzerland

e-mail: jbenthien@uhbs.ch

A. Brunner

Department of Trauma Surgery, Cantonal Hospital Lucerne,

Lucerne, Switzerland articular connections with their neighbouring bones that in case of an osteoarthritic destruction of these articulations may be a cause of considerable pain and rupture of the tendon involved as is described clinically and radiologically $[4,5]$.

Sesamoid bones facilitate the physiological motion of the tendon acting as hypomochlions to ensure correct biomechanical translation [2]. The patella is the most commonly known sesamoid bone in the knee joint. The fabella is also an accessory bone typically and frequently found in the dorsal compartment of the knee joint. It occurs in the context of a fabella complex located in the lateral gastrocnemius muscle and may be bony, cartilagenous or both [6].

Some of the sesamoid bones occurring and being symptomatic in primates and other animals may also be persistent in humans. One of these accessory bones in the knee joint is the cyamella. It is very rare in humans and rarely described in the literature [9-11]. Pathology occurring in humans is infrequently and rarely described and seems to be connected with pathology of the popliteal tendon [9, 11]. The popliteal muscle is a triangularly shaped muscle. It originates from the lateral condyle of the femur and extends in a strong, ca. 4-5-cm-long tendon to insert in the proximal dorsal tibia and assists in internal tibial rotation and knee flexion [12]. A cyamella's displacement associated with rupture of the cruciate ligaments in dogs is described [8].

A cyamella may perform a small articulation with the lateral dorsal femoral condylus $[10,11]$ that may be symptomatic in osteoarthritis [10].

We introduce a case with posterolateral knee pain where a cyamella has incidentally been found in plain radiographs and MRI scan, and some of the patient's pain may be referred to the cyamella. The differential diagnosis to other 
sesamoid bones, namely the fabella, and other foreign bodies, such as osteochondral flakes and periosseous calcifications, is discussed.

\section{Case report}

A 25-year-old male patient presented with a history of posterolateral knee pain and recurrent sensation of swelling and discomfort. These problems occurred gradually about 6 weeks prior to the appointment in our clinic. He has been active in recreational football and jogging prior to our appointment, and his profession was office work. No actual or previously sustained trauma was recorded. Otherwise, his history was unremarkable.

The knee examination yielded no effusion, stable ligaments, negative meniscal signs, some pain on pressure at the lateral knee compartments and some posterolaterally referred pain in knee flexion and internal rotation of the tibia. Range of motion was extension/flexion 0/0/160 degrees, the valgus stress test was negative, the varus stress test was moderately painful.

Antero-posterior and lateral X-rays were performed (Fig. 1a, b). A round osseous structure may be discerned in the posterolateral part of the joint. MRI images were additionally conducted (Fig. 2a, b). No pathological results were obtained apart from a clearly visible ovoid-shaped bone that was seen posterior and superior located near the proximal musculo-tendinous intersection of the popliteus muscle. The sesamoid bone articulates non-cartilagenously with the lateral dorsal femoral condyle (Fig. 2a). The signal intensity of the marrow was similar to that of the adjacent bones, and a cortical structure could also been established. These results point to a sesamoid bone rather than an osteochondral flake, a loose body or a periosseous calcification. The diagnosis of a cyamella should be given. The popliteus muscle and tendon were otherwise normal, and no signs of inflammation or laceration could be seen.

The patient was referred to physical therapy, as no operative interventions were planned at this time. Weight bearing limited by pain was permitted. Passive and active physical therapy, muscle strengthening of the extensors and flexors of the thigh were performed. Specific stretching exercises were not recommended. Laser therapy did not seem to be an option, as the cyamella is not easily accessible for a laser. A follow-up after 1 year demonstrated a completely asymptomatic patient.

\section{Discussion}

The accessory sesamoid bones of the knee seem to have a correlation to phylogenetic development, as their frequency diminishes in higher primates and humans $[3,7]$. They seem to be under substantial genetic control. Early appearance in evolution is seen in Jurassic fossils [3].

Unusual calcified structures on joint imaging may be seen on several occasions. On plain X-rays, naturally only radiologically dense objects like sesamoid bones or osteochondral flakes are seen, whereas in MRI scans,
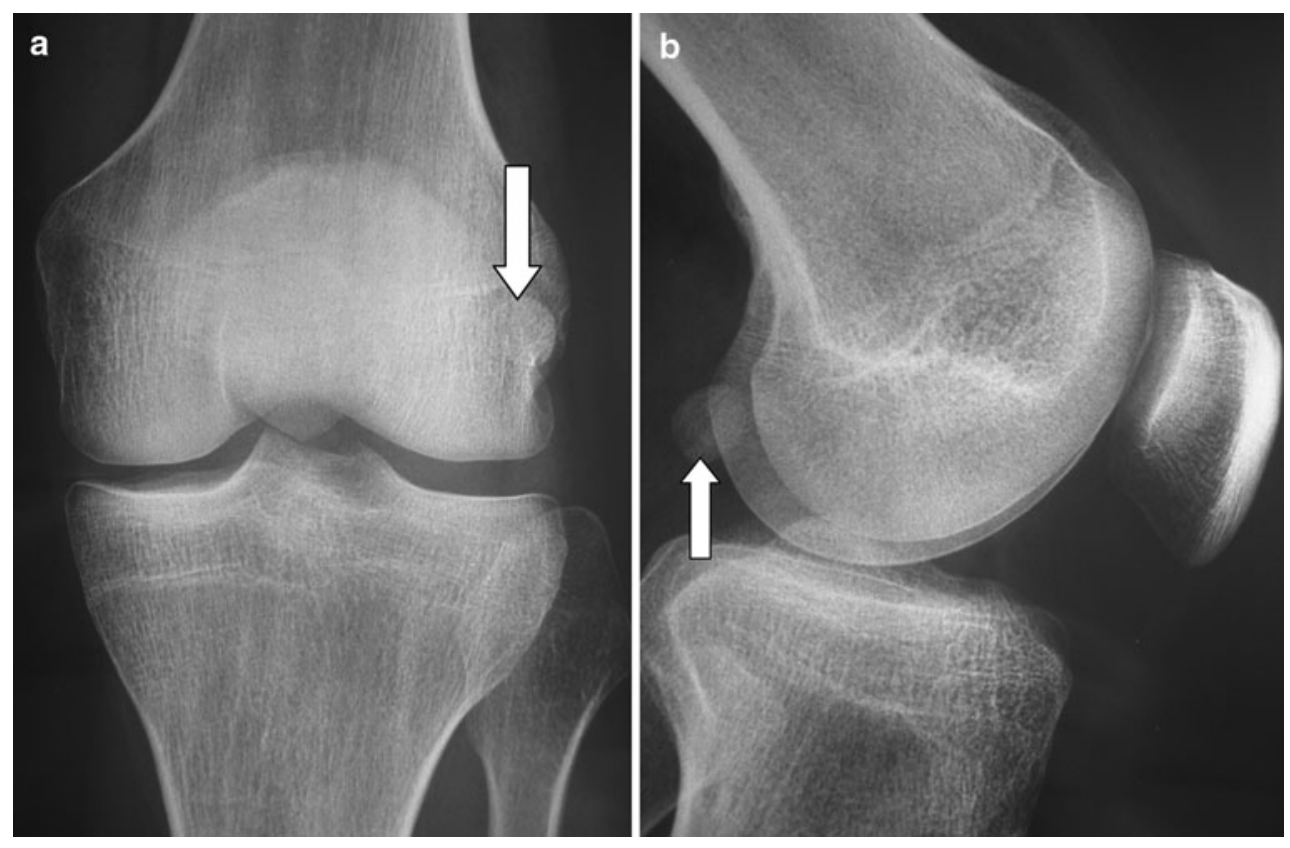

Fig. 1 a a.p. view of the left knee. The cyamella may be seen latero-superior (arrow) as a round osseous structure. $\mathbf{b}$ lateral view of the left knee demonstrating the cyamella as a round osseous body (arrow) 

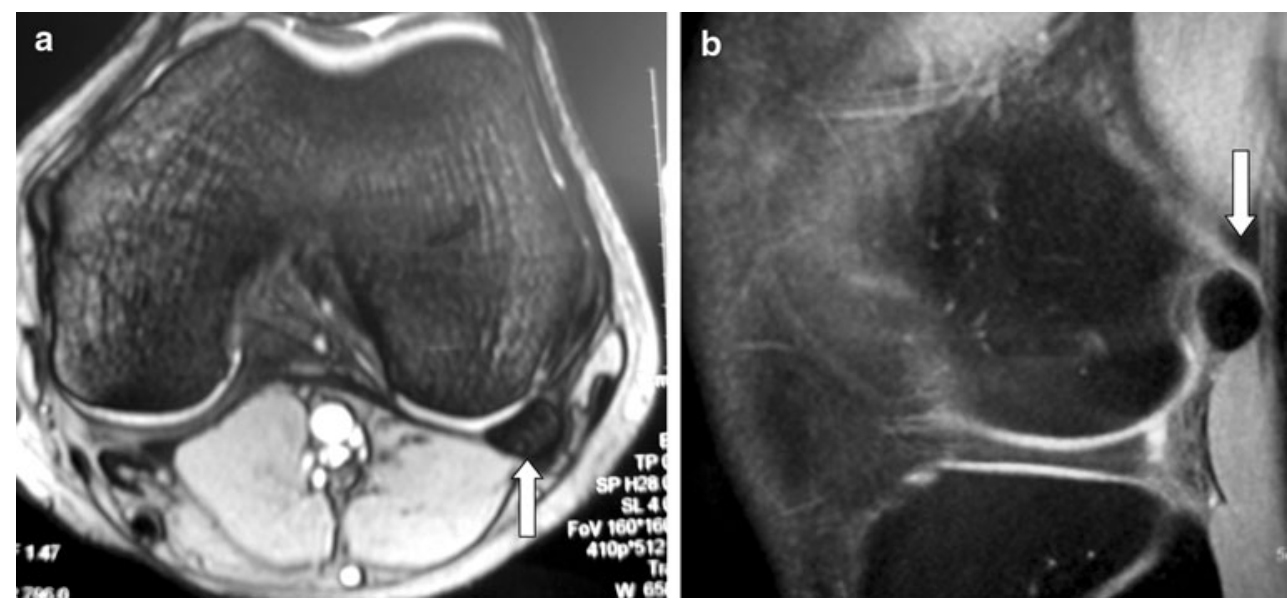

Fig. 2 a MRI scan of the left knee. The cyamella is shown with cortical and cancellous parts in the popliteal tendon posteriorsuperior. An articulation between the cyamella and the posterior

femoral condylus may be seen. Note that cartilage is missing on the cyamella's surface. b The cyamella (arrow) is shown embedded in the popliteal tendon

chondral structures may be discerned. Sesamoid bones typically form complete bony structures with round cortical structures that may be clearly distinguished from fracture flakes or osteophytes $[1,2,10,11]$. MRI imaging for sesamoid bones demonstrates mostly round osseous structures with usually clearly distinctive cortical and cancellous bone $[10,11]$. Osteochondral flakes usually occur intraarticulary, rarely at the periphery of the joint when they may have dislodged into the synovia. They should not occur extraarticulary or in conjunction with tendons. These would be features typically associated with sesamoid bones.

Periarticular or periosseus calcification may be idiopathic or due to trauma (operative procedures, fractures, trauma to the central nervous system). They are almost entirely found outside the joint space and usually do not have a distinction between cortical and cancellous bone [1]

A symptomatic cyamella in humans is very rare and described in the literature only in case reports [9-11]. Remarkable is the difference in size of cyamellae as reported in the literature [9-11]. Mishra and Jurist [9] report a symptomatic cyamella in a 26 -year-old male after a recreational sports-related injury with similar symptoms to our patient. Possible popliteal tendon luxation around the cyamella is discussed in this article. We could not find this in our patient.

Clinical symptoms that may reflect popliteal tendon pathology and possible subluxation around the cyamella were present in our patient in a pain that presented with flexion and internal rotation of the tibia. The symptoms described by Munk et al. [10] were different: chronic knee pain with instability and locking is described. These are features that we would not necessarily associate with a symptomatic cyamella, as they are unspecific and would not apply to pathology of the popliteus tendon.

We decided against operative treatment in our patient, as the removal of this accessory bone would probably weaken the popliteal tendon considerably and diminish its function. Symptomatic cyamellae in patients with osteoarthritis may require an operative removal, but in the only case we found in the literature [10] no treatment option is given. The question whether operative removal should be performed remains an individual decision by the surgeon and cannot be answered considering the few cases described in the literature.

The conservative treatment was effective as the patient as asymptomatic after 3 months of conservative treatment. He remained asymptomatic after a 1-year follow-up.

\section{Conclusion}

The cyamella is a rare sesamoid bone of the popliteus tendon. It may be difficult to diagnose, but it may also be cause of pain related to the popliteal tendon. No specific treatment options are given in the literature, but the few articles treating the subject seem to point towards conservative treatment. The symptoms described in the literature seem to be non-specific.

\section{References}

1. Kransdorf MJ, Peterson JJ, Bancroft LW (2007) MR imaging of the knee: incidental osseous lesions. Radiol Clin N Am 45:943-954

2. Leventen EO (1991) Sesamoid disorders and treatment. An update. Clin Orthop Relat Res 269:236-240

3. Sarin VK, Erickson GM, Giori NJ, Bergmann AG, Carter DR (1999) Coincident development of sesamoid bones and clues to their evolution. New Anat 257:174-180 
4. Sanders TG, Rathur SK (2008) Imaging of painful conditions of the hallucal sesamoid complex and plantar capsular structures of the first metatarsophalangeal joint. Radiol Clin North Am 46(6):1079-1092

5. Valbuena SE, Roulot E (2008) Attrition rupture of the flexor pollicis longus in rheumatoid arthritis on an interphalangeal sesamoid bone. J Hand Surg Eur 33(4):539-540

6. Kawashima T, Takeishi H, Yoshitomi S, Ito M, Sasaki H (2007) Anatomical study of the fabella, fabellar complex and its clinical implications. Surg Radiol Anat 29:611-616

7. LeMinor JM (1992) Brief communication: the popliteal sesamoid bone (cyamella) in primates. Am J Phys Anthropol 87:107-110
8. DeRooster H, van Bree H (1999) Popliteal sesamoid displacement associated with cruciate rupture in the dog. J Small Anim Pract 40:316-318

9. Mishra AK, Jurist KA (1996) Symptomatic cyamella. Arthroscopy 12:327-329

10. Munk PL, Althathol A, Rashid F, Malfair D (2009) MR features of a giant cyamella in a patient with osteoarthritis. Skeletal Radiol 38:69

11. Gur A, Nagihan I, Tahsin H, Yonca A, Sertac A (2006) Popliteus muscle sesamoid bone (cyamella): appearance on radiographs, CT and MRI. Surg Radiol Anat 28:642-645

12. Putz R, Muhlhofer H, Ercan Y (2007) Ligaments of the knee. Orthopade 36(7):612, 614-619 